Draft date July 29,1992

To be submitted for publication in J. Chem. Phys. .

\title{
A global potential energy surface for $\mathrm{ArH}_{2}$
}

\author{
David W. Schwenke, \\ NASA Ames Research Center, MS 230-3, Moffett Field, CA 94035-1000 \\ Stephen P. Walch* and Peter R. Taylor** \\ ELORET Institute, Palo Alto, CA 94303
}

\begin{abstract}
:
We describe a simple analytic representation of the $\mathrm{ArH}_{2}$ potential energy surface which well reproduces the results of extensive $a b$ initio electronic structure calculations. The analytic representation smoothly interpolates between the dissociated $\mathrm{H}_{2}$ and strong bonding limits. In the fitting process, emphasis is made on accurately reproducing regions of the potential expected to be important for high temperature (ca. $3000 \mathrm{~K}$ ) collision processes. Overall, the anisotropy and $\mathrm{H}_{2}$ bond length dependence of the analytic representation well reproduce the input data.
\end{abstract}

* Mailing address: NASA Ames Research Center, MS 230-3, Moffett Field, CA 940351000

** Current address: San Diego Supercomputer Center, P.O. Box 85608, San Diego, CA 92186-9784 


\section{Introduction}

In this paper we present a new potential energy surface (PES) for $\mathrm{ArH}_{2}$. This PES is specifically tailored for use in high temperature ( $c a .3000 \mathrm{~K})$ energy transfer studies involving dissociation and recombination of $\mathrm{H}_{2}$. This potential will be used to help understand the recombination process

$$
\mathrm{H}+\mathrm{H}+\mathrm{M} \rightarrow \mathrm{H}_{2}+\mathrm{M} .
$$

The recombination rates for $\mathrm{M}=\mathrm{H}_{2}$ [1], Ar [2] and $\mathrm{H}_{2} \mathrm{O}$ [2] have been computed as a function of temperature using potential energy surfaces from the literature [3-5]. The result is that within a factor of 1.4 over the temperature range 1000 $5000 \mathrm{~K}$, the recombination rate is independent of the third body $\mathrm{M}$. This contrasts with the prevailing experimental interpretation [6] which assigns the efficiencies 5:1:0.3 for $\mathrm{H}_{2} \mathrm{O}, \mathrm{H}_{2}$ and $\mathrm{Ar}$ at $2000 \mathrm{~K}$. There are several potential sources for this disagreement: inaccuracies in the potential energy surfaces used, limitations in the dynamics/kinetics calculations (see Ref.[1] for a description of the techniques we use to compute the recombination rate given a PES), or experimental uncertainties. Certainly of the three third bodies, the PES for M=Ar was the least well known. In this paper we give a PES of comparable quality to the potential energy surfaces for the other third bodies we have studied.

When constructing a PES, many different strategies are possible. One can combine data from various sources, both theoretical and experimental, and attempt to produce a function which reproduces this information. This procedure is hampered by the possibilities of inconsistency between the various input data. An alternate approach, which is used here, is to just consider data from one source, namely high quality $a b$ initio electronic structure calculations. This strategy can be criticized in that certain features of the PES, such as the weak van der Waals minimum, are obtainable more easily from experimental analysis than from theoretical calculations. However, in our opinion, this drawback is more than counterbalanced by the consistency of the input data which should give rise to a PES which may more accurately reflect the shape of the accurate PES over wide regions of configuration space. We also anticipate that the features of the PES likely to be given most accurately by the electronic structure calculations, namely regions of moderate repulsion, will be predominately responsible for governing the outcome of the high temperature energy transfer processes which are of primary interest to us. 
In fitting a PES for which a wide range of different interactions are possible, one has to choose between a simple function which can be expected to be accurate only in the mean or a more complicated function with many parameters. For a simple function, it is often easy to ensure that it is well behaved in extrapolated regions, while for more complicated functions it may be very difficult to ensure that extrapolation to geometries not included in the fitting procedure will give rise to physically reasonable results. In the present work we begin by using a very simple function to represent the $\mathrm{Ar}+\mathrm{H}_{2}$ interaction and then carefully modify it to ensure that it is well behaved globally yet accurately reproduces the input data. This is achieved using only eight parameters optimized to fit 71 energy points.

\section{Electronic structure calculations.}

The Ar basis set starts with a (17s12p6d4f) primitive set. The two outermost s, $p$, and $d$ and the outermost $f$ primitives are left uncontracted and the inner functions are contracted using an atomic natural orbital (ANO) scheme [7] to [5s4p2d1f]. In addition a (2s2p1d1f) set of even tempered diffuse functions is added. The resulting basis is denoted as $[5+1+1 \mathrm{~s} 4+1+1 \mathrm{p} 2+1+1 \mathrm{~d} 1+1 \mathrm{f}]+(2 \mathrm{~s} 2 \mathrm{p} 1 \mathrm{~d} 1 \mathrm{f})$.

The $\mathrm{H}$ basis set starts with a $(8 \mathrm{~s} 6 \mathrm{p} 4 \mathrm{~d})$ primitive set. The outermost $\mathrm{s}$ and $\mathrm{p}$ primitives are left uncontracted and the inner functions are contracted based on ANO's to [3s2p1d]. This basis is denoted as [3+1s $2+1 \mathrm{p} 1 \mathrm{~d}]$.

The energy calculations were designed to give an accurate description of the process $\mathrm{H}+\mathrm{H}+\mathrm{Ar} \rightarrow \mathrm{H}_{2}+$ Ar. The most important nondynamical electron correlation effects were taken into account by means of complete active space, self consistent field (CASSCF) calculations, and the dynamical correlation effects were estimated using the approximately size-extensive averaged coupled pair functional (ACPF)[8] method. The CASSCF configurations correspond to a single configuration description of $\mathrm{Ar}$ and a two-electron two active orbital description of $\mathrm{H}_{2}$. The full CASSCF reference space was used in the ACPF calculations. All calculations were carried out using the MOLECULE-SWEDEN [9] program system on the Ames Research Center Advanced Computation Facility CRAY Y-MP/864.

The ANO basis for $\mathrm{H}_{2}$ was optimized at the equilibrium bond length $\left(R_{e}\right)$ [7]. This makes the basis set superposition error much smaller at $R_{e}$ than at other $\mathrm{H}_{2}$ bond lengths. To help correct for this systematic error, we corrected all our energies by means of the Boys and Bernardi function counterpoise method[9.1]. Specifically, 
we computed the following energies:

$E^{\mathrm{ArH}_{2}}(G)$, the energy of the $\mathrm{ArH}_{2}$ supermolecule at the ACPF level at geometry $G$,

$E^{\mathrm{H}_{2}}(G)$, the energy of the $\mathrm{H}_{2}$ molecule at the all single and double excitation configuration interaction (SDCI) level at geometry $G$ including the Ar oneelectron basis functions,

$E^{\mathrm{Ar}}(G)$, the energy of the $\mathrm{Ar}$ atom at the ACPF level at geometry $G$ including the $\mathrm{H}$ atom one-electron basis functions.

Let $G^{\infty}$ be a geometry approximating Ar not interacting with $\mathrm{H}_{2}$ but having the same $\mathrm{H}_{2}$ bond length as in geometry $G$. In practice, not interacting means any two atom-atom distances greater than $30 a_{0}$. Then we take the energy of interaction between $\mathrm{Ar}$ and $\mathrm{H}_{2}$ to be

$$
E^{i n t}(G)=E^{\mathrm{ArH}_{2}}(G)-E^{\mathrm{ArH}_{2}}\left(G^{\infty}\right)+E^{\mathrm{H}_{2}}\left(G^{\infty}\right)-E^{\mathrm{H}_{2}}(G)+E^{\mathrm{Ar}_{\mathbf{r}}}\left(G^{\infty}\right)-E^{\mathrm{Ar}}(G)
$$

It should be noted that, since the SDCI and ACPF methods are not exactly sizeextensive, it is important to use Eq.(1) rather than $E^{\mathrm{ArH}_{2}}(G)-E^{\mathrm{H}_{2}}(G)-E^{\mathrm{Ar}}(G)$ to compute the interaction energy since $E^{\mathrm{ArH}_{2}}\left(G^{\infty}\right) \neq E^{\mathrm{Ar}}\left(G^{\infty}\right)+E^{\mathrm{H}_{2}}\left(G^{\infty}\right)$.

In Tables I and II we give the values of $E^{\text {int }}$ computed in the course of this work. The geometries are specified by the Jacobi coordinates $r, R$, and $\chi$, where $r$ is the distance between the $\mathrm{Ar}$ and the center of mass of the $\mathrm{H}_{2}, R$ is the $\mathrm{H}_{2}$ bond length, and $\chi$ is the Ar-center of mass $\mathrm{H}_{2}-\mathrm{H}$ angle.

\section{Fitting the PES}

We will optimize the parameters $(\vec{P})$ in our fitting function $\left[V^{\text {int }}(G, \vec{P})\right]$ by minimizing the least squares function

$$
\phi(\vec{P})=\sum_{i}\left\{\left[E^{i n t}\left(G_{i}\right)-V^{i n t}\left(G_{i}, \vec{P}\right)\right] / w_{i}\right\}^{2}
$$

An important aspect of the present work is the choice of the weights $w_{i}$. Previous work [10] has shown strong correlations between the force along the diatom bond evaluated at the classical turning point for relative translation and vibrational energy transfer rates, thus we wish to concentrate our interest to regions where $V^{\text {int }} \sim E_{\text {rel }}$. At $3000 \mathrm{~K}, k_{\mathrm{B}} T=10^{-2} E_{\mathrm{h}}$. Also $\omega \hbar$ for $\mathrm{H}_{2}$ is about $2 \times 10^{-2} E_{\mathrm{h}}$, so we want to focus in most closely on energies in the range $5-50 \mathrm{~m} E_{\mathrm{h}}$. We will 
identify the weights as an acceptable error in the fit for each point. In the most interesting region, a $5 \%$ error is reasonable, thus we take

$$
w_{i}=\max \left\{\begin{array}{l}
\left\{0.0375\left[\log \left|V^{i n t}\left(G_{i}\right) / E_{\mathrm{h}}\right|+2\right]^{2}+0.05\right\}\left|V^{i n t}\left(G_{i}\right)\right| \\
10^{-4} E_{\mathrm{h}} .
\end{array}\right.
$$

This is a parabola in \% error as a function of the order of magnitude of the energies, centered on $10 \mathrm{~m} E_{\mathrm{h}}$, except at very weakly interacting geometries, where we let the acceptable error be as large as $0.1 \mathrm{~m} E_{\mathrm{h}}$. With this weighting scheme, reporting the root mean square deviation between the fit and the input data is not meaningful - the important quantity is $\phi(\vec{P})$, which is unitless. A value equal to one means that on the average, we have attained our goal in fitting the points.

The first step in our fitting process is the determination of an ArH potential curve, $V^{\mathrm{ArH}}$. We will use this in a zeroth order representation of the $\mathrm{ArH}_{2}$ interaction energy, i.e.

$$
V^{i n t}=V^{\mathrm{ArH}}\left(R_{+}\right)+V^{\mathrm{ArH}}\left(R_{-}\right)
$$

where $R_{ \pm}$are the two ArH distances, i.e.

$$
R_{ \pm}=\left[r^{2}+(R / 2)^{2} \pm r R \cos \chi\right]^{\frac{1}{2}}
$$

This simple form will give physically reasonable results for all geometries provided $V^{\mathrm{ArH}}$ is well behaved. We choose to parameterize the potential curve as

$$
V^{\mathrm{ArH}}(X)=A \exp [-b X]-\frac{C_{6}^{\mathrm{ArH}}}{X^{6}+d^{6}}-\frac{C_{8}^{\mathrm{ArH}}}{\left(X^{4}+d^{4}\right)^{2}}-\frac{C_{10}^{\mathrm{ArH}}}{\left(X^{2}+d^{2}\right)^{5}},
$$

where $C_{6}^{\mathrm{ArH}}-C_{10}^{\mathrm{ArH}}$ are taken from Ref.[11]. The three parameters $A, b$, and $d$ are determined by nonlinear least squares using the weights of Eq.(3) and the data given in Table I. The final value of $\phi$ for this fit is 0.23 with maximum weighted difference of 0.45. The final values of the fitting parameters are given in Table III where they are labeled $A_{0}, b_{0}$ and $d_{0}$. With these parameters, $V^{\mathrm{ArH}}$ is well behaved for all atom-atom distances.

We now turn to geometries where the two $\mathrm{H}$ atoms are interacting with the Ar. We start with the simple sum of pairwise interactions, Eq.(4). This simple form has several deficiencies. We correct for these deficiencies by introducing additional 
dependence on the coordinates $r, R$, and $\chi$ other than that implicit in Eq.(5). In introducing the additional coordinate dependence, one has considerable flexibility. However, since in the limit $R$ goes to $\infty, \mathrm{Eq} .(4)$ is exact, we require that the corrections to $\mathrm{Eq} .(4)$ vanish in this limit. The easiest way to enforce this limit is to have the corrections depend only on $R$ and not on $r$ or $\chi$. We thus considered an intermediate step in the fitting process, namely fitting the $a b$ initio data for fixed bond lengths. This will yield parameters fully optimized as a function of $R$. In the final step, we will fit the dependence of the parameters on $R$. The intermediate step involves fewer points and parameters than the final step and provides excellent initial guesses for nonlinear parameters. It will also tell us whether or not it is possible to obtain a satisfactory fit with only $R$ dependent parameters. In the present case, we will see that it will be possible to obtain accurate fits with this restriction.

The first correction to Eq.(4) concerns the long range attractive term. The leading contribution from $C_{6}^{\mathrm{ArH}} /\left(R_{ \pm}^{6}+d^{6}\right)$ expanded in terms of the Jacobi coordinates is $2 C_{6}^{\mathrm{ArH}} / r^{6}+O\left(r^{-8}\right)$ which can be compared with the more accurate form $C_{6}^{\mathrm{ArH}_{2}}(R)\left[1+\Gamma_{6}^{\mathrm{ArH}_{3}}(R) P_{2}(\cos \chi)\right] / r^{6}+O\left(r^{-8}\right)$. Our hypothesis requires that we neglect the $P_{2}$ term, however this is not a bad approximation since $\Gamma_{6}^{\mathrm{ArH}_{2}}(R) \sim 10 \%$ for $R$ near $R_{e}$ [13]. We thus scale the long range part of Eq.(4) by the factor $g(R)=C_{6}^{\mathrm{ArH}_{2}}(R) / 2 C_{6}^{\mathrm{ArH}}$ and we fit the data of Varandas [13] to $g(R)=\left\{\beta \exp \left[-\alpha\left(R-R_{0}\right)\right]+1\right\}^{-1}$ with $\alpha=2 a_{0}^{-1}, \beta=0.437$ and $R_{0}=1.449 a_{0}$. As $R$ becomes large, $g(R)$ becomes unity and we recover the ArH long range interaction.

The next deficiency we address is the anisotropy of the sum of pairwise interactions, e.g. the $\chi$ dependence. Using Eq.(4) with the scaled long range potential results in too much anisotropy. We interpret this as a manifestation of the migration of charge density toward the center of the $\mathrm{H}_{2}$ bond, thus we introduce the shift $\rho$, i.e. we replace $R_{ \pm}$in Eq.(5) with

$$
\tilde{R}_{ \pm}=\left[r^{2}+\left(\frac{R-\rho}{2}\right)^{2} \pm r(R-\rho) \cos \chi\right]^{\frac{1}{2}}
$$


thus we take

$$
\begin{aligned}
& V^{i n t}(r, R, \chi, A, b, d, \rho)=A\left[\exp \left(-b \tilde{R}_{+}\right)+\exp \left(-b \tilde{R}_{-}\right)\right] \\
& -g(R)\left\{C_{6}^{\mathrm{ArH}}\left[\left(\bar{R}_{+}^{6}+d^{6}\right)^{-1}+\left(\tilde{R}_{-}^{6}+d^{6}\right)^{-1}\right]+C_{8}^{\mathrm{ArH}}\left[\left(\tilde{R}_{+}^{4}+d^{4}\right)^{-2}+\left(\bar{R}_{-}^{4}+d^{4}\right)^{-2}\right]\right. \\
& \left.+C_{10}^{\mathrm{ArH}}\left[\left(\bar{R}_{+}^{2}+d^{2}\right)^{-5}+\left(\bar{R}_{-}^{2}+d^{2}\right)^{-5}\right]\right\} .
\end{aligned}
$$

Finally we optimize the four parameters $A, b, d$, and $\rho$ for each value of $R$. The value of $\phi$ for each value of $R$ is given in Table IV under the column marked optimum. A satisfactory fit is obtained for all $\mathrm{H}_{2}$ bond lengths.

To complete our fit, we must parameterize $A(R), b(R), d(R)$, and $\rho(R)$. We first consider $\rho(R)$. Since this parameter is based upon the idea of charge flow, we will tie it to a molecular property which reflects this, namely the quadrupole moment. Specifically we take

$$
\rho(R)=R\left\{1-\left[1+q(R) / \delta R^{2}\right]^{-\frac{1}{2}}\right\}
$$

where $q(R)$ is the quadrapole moment of $\mathrm{H}_{2}$. We will use the fit of Ref.[3] to generate $q(R)$ at all $R$. This contains the single parameter $\delta$. Originally, we tried to parameterize $\rho(R)$ as the displacement of a fixed point charge required to reproduce $q(R)$, but we were not successful. Equation (9) represents the displacement required to reproduce $q(R)$ for a $R$ dependent point charge with $R$ dependence leading to a constant discriminant in the quadratic equation for which $\rho(R)$ is a root.

The remaining functions are represented as an expansion in terms of even tempered exponentials, i.e.

$$
X(R)=\sum_{i=0}^{i_{\max , x}} X_{i}\left[-\exp \left(\alpha_{X} R\right)\right]^{i}
$$

where $X=A, b$, or $d$, with the $i=0$ coefficient fixed by the fit to the $\operatorname{Ar} H$ interaction. In our final fit we use $i_{\max , A}=2$ and $i_{\max , b}=i_{\max , d}=1$. The parameters for the fit are given in Table III and the weighted errors as a function of $R$ in Table IV. The maximum weighted error is 2.7 and the number of geometries with weighted errors greater than one is five. With these parameters, the fit seems to be globally well behaved.

IV. Discussion 
We first consider the ArH interaction. The fit is quite good, however it does not well reproduce the empirical ArH potential from Ref.[11]. See Fig. 1. The present $\mathrm{ArH}$ potential curve has van der Waals minimum parameters $R_{\min }=7.13 a_{0}$ $V_{\min }=-0.102 \mathrm{~m} E_{\mathrm{h}}$ compared to $R_{\min }=6.68 a_{0} V_{\min }=-0.175 \mathrm{~m} E_{\mathrm{h}}$ for the TangToennies potential including up to $C_{18}$. The Tang-Toennies potential is also much more repulsive at distances less than $R_{\min }$. The reason for this discrepancy is not clear, for improving the theoretical calculations by both expanding the one-electron basis set and changing the electron correlation treatment does not significantly change the present result [12].

We can also compare to empirically determined values of the functions in the expansion

$$
V^{i n t}\left(r, R=1.449 a_{0}, \chi\right)=v_{0}(r)+P_{2}(\cos \chi) v_{2}(r)
$$

given in Ref. [14]. This is done in Fig. 2 where we also give $v_{0}$ and $v_{2}$ computed from the $a b$ initio data and the current fit. These functions for the fit do not change significantly between $R=1.401$ and $R=1.449$, so it is meaningful to compare the functions for the two distances. The comparison between the $v_{0}$ term is very similar to the ArH potential: the fit well reproduces the $a b$ initio data and the minimum for the Tang-Toennies function is deeper and closer in: $R_{\min }=6.77 a_{0}$ and $V_{\min }=-0.232 \mathrm{~m} E_{\mathrm{h}}$ versus $R_{\min }=7.10 a_{0}$ and $V_{\min }=-0.157 \mathrm{~m} E_{\mathrm{h}}$ for the fit. In contrast, for the $v_{2}$ term, the Tang-Toennies function fits the $a b$ initio data better than the fit in the well region. The failure of the fit to reproduce the $a b$ initio result is presumably due to the fact that the leading long range anisotropic contribution is proportional to $r^{-8}$. However, since $v_{2}$ is not large, the absolute magnitudes of the deviations are not large.

Now consider the consequence of stretching the $\mathrm{H}_{2}$ bond. The most interesting orientation to consider is that for $\chi=\pi / 2$, the T-shaped geometries. In Fig. 3 we compare the fit to the $a b$ initio data for repulsive geometries. On the whole we see very good agreement between the fit and the data. This particular orientation is interesting because the $a b$ initio data and the fit predict that at $R_{e}, V^{i n t}$ will increase as $R$ increases whereas a simple pairwise additive model would give the reverse trend. It is the inclusion of the $R$ dependent shift $\rho(R)$ which is primarily responsible for the correct sign of the slope for the present fit. Eventually as $R$ increases, the slope changes sign and the fit appears to do a reasonable job at predicting the position of the sign change. However, in this figure, larger deviations appear for 
$R=3$ than for the other geometries. This is also shown by the weighted errors in Table IV. This is probably an indication that a more sophisticated treatment of the anisotropy is required.

In the course of the present work we considered several variants of the present fit. All of these were attempts to improve the description of the anisotropy of the PES. An obvious place to start is to replace the function $g(R)$ used to scale the dispersion terms with the function of Ref.[13] which includes $\chi$ dependence and a more accurate $R$ dependence. We also considered introducing $P_{2}(\cos \chi)$ anisotropy into the functions $A(R), b(R), d(R)$ and $\delta(R)$. By doing so, it was possible to substantially reduce the weighted errors for $R=1$ and 1.401 , but the improvement for $R=3$ and 5 was much more modest. These fits also yielded more complicated $R$ dependence for $A(R)$ etc and so it was very hard to produce functions which gave rise to a globally well behaved PES. On the whole, the present fit was outstanding in its ability to accurately predict the global shape of the PES.

IV. Conclusions

In this paper we have presented a simple yet accurate function to interpolate/extrapolate the interaction energy of $\mathrm{Ar}+\mathrm{H}_{2}$. In conjunction with an accurate $\mathrm{H}_{2}$ potential curve, such as that from Ref.[3], this defines a global PES suitable for studying energy transfer and dissociative collisions. The function does not well reproduce empirical estimates of the van der Waals well region, but does reproduce reasonably well the $a b$ initio data in that region. The force along the $\mathrm{H}_{2}$ bond is well represented globally without recourse to switching functions.

\section{Acknowledgements}

SPW is supported by NASA grant no. NCC2-478 and PRT is supported by NASA grant no. NCC2-371. 
References.

1. D. W. Schwenke, J. Chem. Phys. 92, 7267 (1989).

2. D. W. Schwenke, unpublished calculations.

3. D. W. Schwenke, J. Chem. Phys. 89, 2076 (1988).

4. N. C. Blais and D. G. Truhlar, J. Chem. Phys. 65, 5335 (1976).

5. D. W. Schwenke, S. P. Walch, and P. R. Taylor, J. Chem. Phys 94, 2986 (1991).

6. N. Cohen and K. R. Westberg, J. Phys. Chem. Ref. Data 12, 531 (1983).

7. J. Almlöf and P. R. Taylor, J. Chem. Phys. 86, 4070 (1987).

8. R. J. Gdanitz and R. Ahlrichs, Chem. Phys. Lett. 143, 413 (1988).

9. MOLECULE-SWEDEN is an electronic structure program system written by J. Almlöf, C. W. Bauschlicher, M. R. A. Blomberg, D. P. Chong, A. Heiberg, S. R. Langhoff, P.-Å. Malmqvist, A. P. Rendell, B. O. Roos, P. E. M. Siegbahn, and P. R. Taylor.

9.1 S. F. Boys and F. Bernardi, Mol. Phys. 19, 553 (1970).

10. D. W. Schwenke and D. G. Truhlar, J. Chem. Phys. 81, 5586 (1984).

11. K. T. Tang and J. P. Toennies, Chem. Phys. 156, 413 (1991).

12. H. Partridge, unpublished calculations.

13. A. J. C. Varandas and M. A. Matias, Chem. Phys. Lett. 148, 149 (1988).

14. K. T. Tang and J. P. Toennies, J. Chem. Phys. 74, 1148 (1981). 
Table I. ArH interaction energies.

\begin{tabular}{rrr}
\hline \hline$R_{\mathrm{ArH}}\left(a_{0}\right)$ & $E^{i n t}\left(\mathrm{~m} E_{\mathrm{h}}\right)$ & $V^{\mathrm{ArH}}\left(\mathrm{m} E_{\mathrm{h}}\right)$ \\
\hline \hline 2. & 215.740 & 205.625 \\
3. & 50.853 & 49.295 \\
4. & 11.278 & 11.505 \\
5. & 1.912 & 1.885 \\
6. & 0.119 & 0.110 \\
7. & -0.095 & -0.101 \\
8. & -0.072 & -0.078 \\
9. & -0.040 & -0.044 \\
10. & -0.021 & -0.024 \\
\hline
\end{tabular}


Table II. $\mathrm{ArH}_{2}$ interaction energies.

\begin{tabular}{rrrrr}
\multicolumn{5}{c}{$E^{\text {int }}\left(\mathrm{m} E_{\mathbf{h}}\right)$} \\
$r\left(a_{0}\right)$ & $R=1 a_{0}$ & $R=1.401$ & $R=3$ & $R=5$ \\
\cline { 2 - 5 } 3. & 158.061 & 182.810 & 535.157 & $a$ \\
4. & 25.707 & 32.480 & 73.251 & 522.077 \\
5. & 3.202 & 4.546 & 16.575 & 90.337 \\
6. & 0.089 & 0.219 & 2.757 & 22.709 \\
8. & -0.094 & -0.129 & -0.218 & 0.533 \\
10. & -0.025 & -0.035 & -0.082 & -0.103 \\
& & $\chi=\pi / 4$ & & \\
3. & 144.256 & 154.744 & 151.256 & 150.373 \\
4. & 23.772 & 28.272 & 41.471 & 62.092 \\
5. & 2.981 & 3.937 & 8.873 & 18.126 \\
6. & 0.098 & 0.190 & 1.175 & 4.016 \\
8. & -0.082 & -0.112 & -0.185 & -0.088 \\
10. & -0.020 & -0.031 & -0.062 & -0.068 \\
& & & & \\
3. & 133.271 & 136.783 & 97.310 & 29.719 \\
4. & 22.015 & 24.734 & 23.799 & 8.183 \\
5. & 2.765 & 3.404 & 4.233 & 1.444 \\
6. & 0.097 & 0.164 & 0.340 & -0.016 \\
8. & -0.077 & -0.097 & -0.145 & -0.119 \\
10. & -0.021 & -0.027 & -0.045 & -0.037 \\
& & & & \\
\hline \hline
\end{tabular}

${ }^{a}$ Very repulsive so not computed. 
Table III. $\mathrm{ArH}_{2}$ PES parameters (in atomic units).

\begin{tabular}{rrrrr}
\hline \hline & & & & \\
$\mathrm{X}$ & $X_{0}$ & $X_{1}$ & $X_{2}$ & $\alpha_{X}$ \\
\hline \hline$A$ & 18.06 & 58.63 & -90.61 & 0.7662 \\
$b$ & 1.653 & 0.3874 & & 0.7662 \\
$d$ & 2.339 & & & \\
& & & $\delta=0.2369$ & \\
\hline
\end{tabular}


Table IV. Root mean square errors for $\mathrm{ArH}_{2}$ PES fit.

\begin{tabular}{crc}
\hline \hline & & \\
$R$ & Optimum & Fit \\
\hline \hline 1.0 & 0.20 & 0.32 \\
1.401 & 0.29 & 0.46 \\
3.0 & 0.85 & 0.98 \\
5.0 & 0.55 & 0.82 \\
all & 0.54 & 0.70 \\
& & \\
\hline \hline
\end{tabular}




\section{Figure captions:}

Figure 1: The ArH potential curve from $a b$ initio calculations (symbols), from the present fit , Eq. (6) (solid line), and from Tang and Toennies [11]. Note the switch between a linear and $\log$ scale at $0.1 \mathrm{~m} E_{\mathrm{h}}$.

Figure 2: The first two terms in a Legendre expansion of the $\mathrm{ArH}_{2}$ PES. Circles, $v_{0}$ from $a b$ initio points; squares $v_{2}$ from $a b$ initio points; solid line fit; dashed line, the fit of Tang and Toennies [14]. Note the switch between a linear and log scale at $0.1 \mathrm{~m} E_{\mathrm{h}}$.

Figure 3: The dependence of the potential energy on the $\mathrm{H}_{2}$ bond length for $\chi=$ $\pi / 2$. The symbols are the $a b$ initio points with squares for $r=3 a_{0}$, circles for $r=4$, triangles for $r=5$, and crosses for $r=6$. The solid lines are the fit at the corresponding values of $r$. 


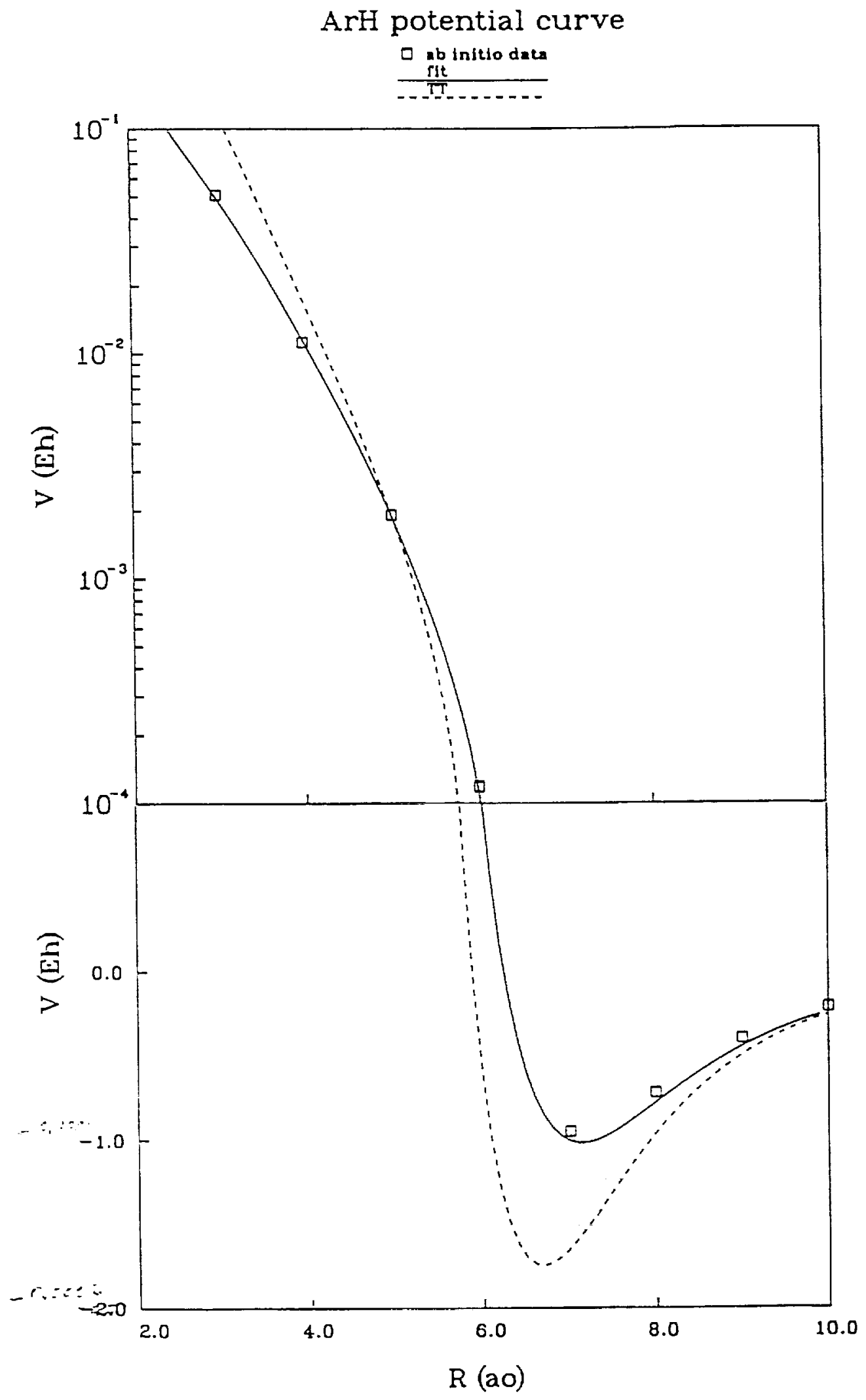

fig. 1 


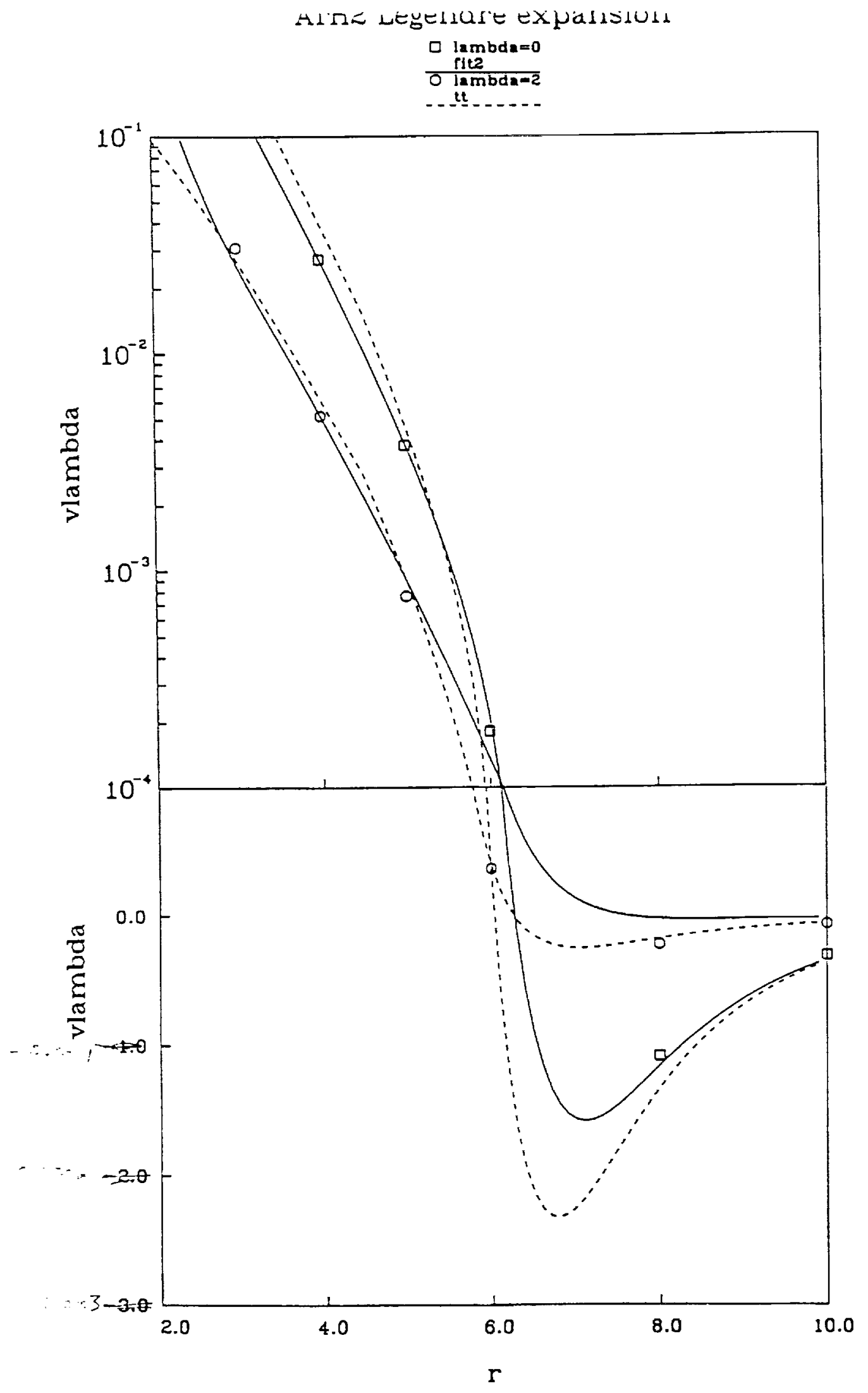

fig. 


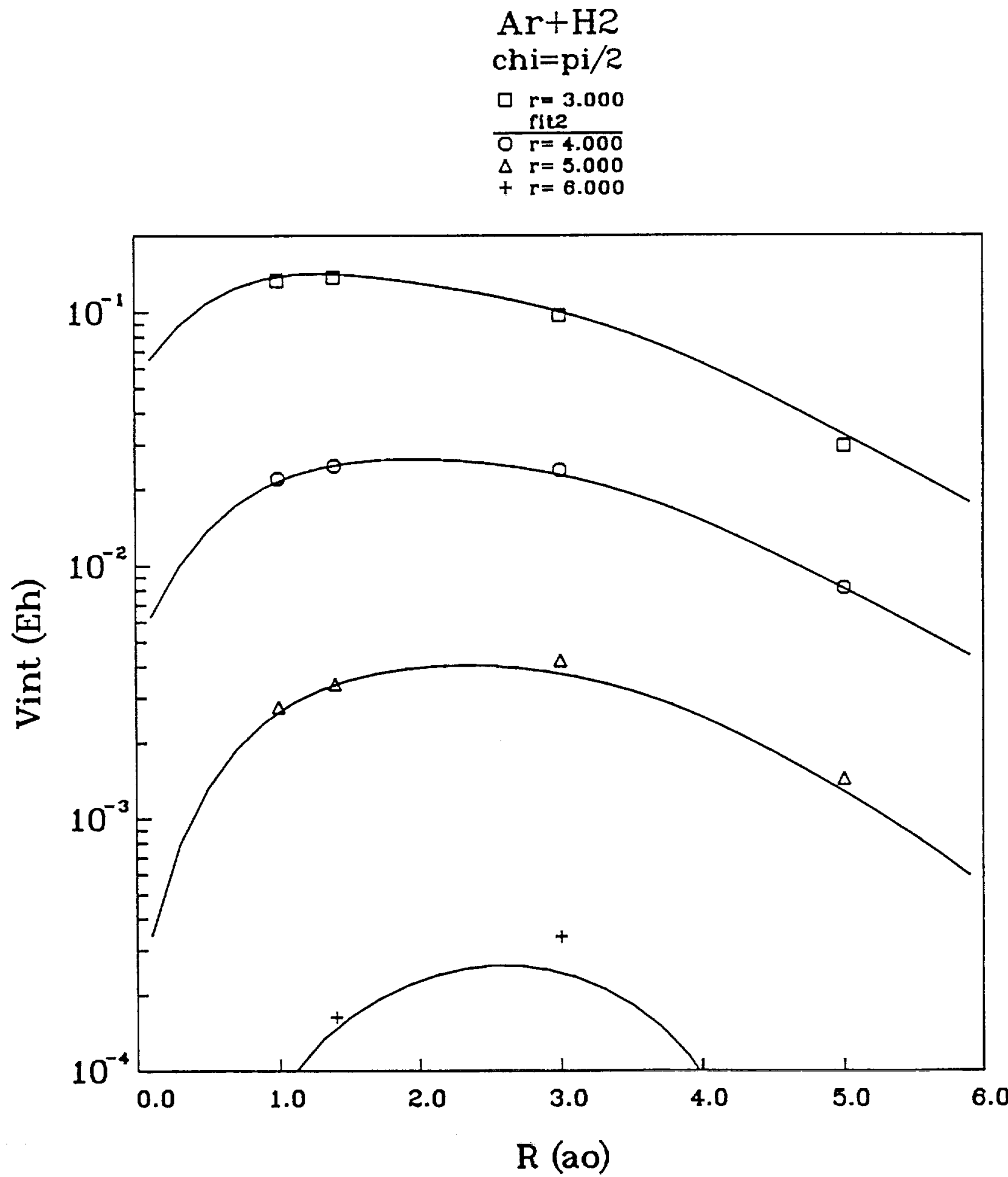

fig. 3 